\title{
Workshop on Computational Advances in Molecular Epidemiology
}

\author{
Yury Khudyakov \\ Division of Viral Hepatitis \\ Centers of Disease Control \\ and Prevention \\ Atlanta, GA 30329 \\ yek0@cdc.gov
}

\author{
Ion Mandoiu \\ Computer Science \& \\ Engineering Department \\ University of Connecticut \\ Storrs, CT 06269 \\ ion@engr.uconn.edu
}

\author{
Pavel Skums \\ Department of Computer \\ Science \\ Georgia State University \\ Atlanta, GA 30303 \\ pskums@gsu.edu
}

\author{
Alexander Zelikovsky \\ Department of Computer \\ Science \\ Georgia State University \\ Atlanta, GA 30303 \\ alexz@cs.gsu.edu
}

Welcome to the $8^{\text {th }}$ Workshop on Computational Advances in Molecular Epidemiology, held on September 7, 2019 in Niagara Falls, NY in conjunction with the $10^{\text {th }} A C M$ Conference on Bioinformatics, Computational Biology, and Health Informatics $(A C M-B C B){ }^{1}$

Molecular epidemiology is essentially an integrative scientific discipline that considers molecular biological processes in specific epidemiological settings. It relates molecular biological events to etiology, distribution and prevention of disease in human populations. Over years, molecular epidemiology has become extensively fused with mathematical and computational science and immensely benefitted from this tight association. The CAME workshop aims to provide a forum for presentation and discussion of the latest computational research in molecular epidemiology. This multidisciplinary workshop brings together field practitioners of molecular epidemiology, molecular evolutionists, population geneticists, medical researchers, bioinformaticians, statisticians and computer scientists interested in the latest developments in algorithms, mining, visualization, modeling, simulation and other methods of computational, statistical and mathematical analysis of genetic and molecular data in the epidemiological context.

This year's workshop builds upon 7 prior editions successfully held in Atlanta (Nov. 12-15, 2011, in conjunction with the 2011 IEEE International Conference on Bioinformatics and Biomedicine), New Orleans (June 12-14, 2013, in conjunction with the $3^{\text {rd }}$ IEEE International Conference on Computational Advances in Bio and Medical Sciences), Miami Beach (June 2-4, 2014 , in conjunction with the $4^{\text {th }}$ IEEE International Conference on Computational Advances in Bio and Medical Sciences), Norfolk, Virginia (June 7-10, 2015, in conjunction with the $11^{\text {th }}$ International Symposium on Bioinformatics Research and Applications), Atlanta (October 13-15, 2016, in conjunction with the $6^{\text {th }}$ IEEE International Conference on Computational
Advances in Bio and Medical Sciences), Boston (August 20, 2017 in conjunction with the 8 th ACM Conference on Bioinformatics, Computational Biology, and Health Informatics), and Washington, D.C. (August 29, 2018 in conjunction with the $9^{\text {th }} A C M$ Conference on Bioinformatics, Computational Biology, and Health Informatics).

This year the workshop program includes 15 invited talks that cover the following topics:

- computational methods for viral transmissions inference and molecular surveillance, including phylogenetic and phylodynamics methods for inference of transmission histories and outbreak response, development of computational tools and platforms for epidemiological networks construction and analysis, applications of analysis of genomic data for outbreak investigation.

- Study of intra-host viral evolution, including algorithms for reconstruction of intra-host viral populations from Nextgeneration sequencing data, inference of fitness landscapes of heterogeneous populations, differentiation between recent and chronic infections, study of viral resistance and immune adaptation.

- computational methods for immunogenomics and cancer genomics, including an inference and prediction of cancer evolutionary and progression history, identification of epitopes and immunodiagnostics.

Speakers will be invited to submit full length articles to special issue of BMC Bionformatics.

We would like to thank BCB Workshop Co-chairs Wei Zhang and Xia Ning for the opportunity to organize CAME 2019 in conjunction with the 10th ACM-BCB. Finally, we would like to thank all speakers for presenting their work at the workshop.

\footnotetext{
${ }^{1}$ Permission to make digital or hard copies of part or all of this work for personal or classroom use is granted without fee provided that copies are not made or distributed for profit or commercial advantage and that copies bear this notice and the full citation on the first page. Copyrights for third-party components of this work must be honored. For all other uses, contact the Owner/Author.

$A C M-B C B$ '19, September 7-10, 2019, Niagara Falls, NY, USA

(C) 2019 Copyright is held by the owner/author(s)

ACM ISBN 978-1-4503-6666-3/19/09.

https://doi.org/10.1145/3307339.3343859
} 